\title{
Monitoring Forests: A New Paradigm of Remote Sensing \& GIS Based Change Detection
}

\author{
Subhash Ashutosh \\ Indira Gandhi National Forest Academy (IGNFA), Dehradun, India \\ Email: sashutosh30@yahoo.com
}

Received July 23, 2012; revised August 21, 2012; accepted September 21, 2012

\begin{abstract}
Remote sensing has emerged as the main tool for mapping and monitoring of forest resources globally. In India, this technological tool is in use for biennial monitoring of forest cover of the country for the last 25 years. Among the numerous applications of remote sensing in forest management, change detection is the one which is most frequently used. In this paper, a new paradigm of change detection has been presented in which change of vegetation in a grid (a square shaped unit area) is the basis of change analysis instead of change at the pixel level. The new method is a simpler approach and offers several advantages over the conventional approaches of remote sensing based change detection. The study introduces an index termed as "Grid Vegetation Change Index (GVCI)", its numerical value gives quantified assessment of the degree of change. The minus value of GVCI indicates loss or negative change and similarly positive value vice versa. By applying the GVCI on a pair of remotely sensed images of two dates of an area, one can know degree of vegetation change in every unit area (grid) of the large landscape. Based on the GVCI values, one can select those grids which show significant changes. Such "candidate grids with significant changes" may be shortlisted for ground verification and studying the causes of change. Since the change identification is based on the index value, it is free from human subjectivity or bias. Though there may be some limitations of the methodology, the GVCI based approach offers an operational application for monitoring forests in India and elsewhere for complete scanning of forest areas to pointedly identify change locations, identifying the grids with significant changes for objective and discrete field inspections with the help of GPS. It also offers a method to monitor progress of afforestation and conservation schemes, monitor habitats of wildlife areas and potential application in carbon assessment methodologies of CDM and REDD+.
\end{abstract}

Keywords: Change Detection; Grids; GVCI; Zonal Statistics

\section{Introduction}

India has 69.20 million ha of forest cover according to the latest assessment done by FSI (ISFR 2011) [1] With over 170,000 villages located in proximity of forests and given the rising human and livestock population in these villages, there is intense anthropogenic pressure on most of the country's forests. India is also witnessing a phase of rapid economic growth which has two fold implication on its forest resources, first, the demand of wood and other forest based products is rising with the rising income of people and secondly the increasing requirement of land for infrastructure development and mining etc is exerting pressure for diversion of forests in many areas. Conservation of forests in this scenario is a great challenge, whereas, at the same time it is of paramount importance from the ecological security point of view and for ensuring sustained growth and food security in the country.

Intensive monitoring of forests is the first and fore- most requirement for conservation and sustainable management of forest resources. Considering the challenges of forest protection in the current times, it is necessary that the available technological options should be tried and if practicable, should be employed to give an edge to the conventional field patrolling system which we have in the Departments for protecting forests. An effective monitoring system applicable over vast tract of forests should have the following essential elements viz 1) it should be periodic with possible frequent monitoring in short span of time; 2) it should be complete i.e. it should scan the whole area for detecting changes and no change (above a threshold) should go undetected; 3 ) it should not be cost prohibitive; 4) it should be technologically implementable as an operational system in the given set up of the organization (Forest Departments).

An increasing requirement of close monitoring of forests and ecologically sensitive areas has been felt in the recent times both at the level of policy and planning and 
more at the level of field managers for an early detection of degradation or damage caused to the forest ecosystems so that quick measures for arresting the degradation process could be taken. There is also a long felt requirement of robust system for monitoring progress of various center and State funded schemes of conservation and afforestation being executed in the field. Intensive monitoring of wildlife habitats is another important requirement for their effective protection. An effective monitoring system brings in transparency in the system and is essential for improved governance.

Remote sensing based monitoring of forests due to its capability of synoptic coverage of vast areas is in vogue for at least three decades now and is well established practice in many countries. In India, FSI is monitoring forest cover of the country using remote sensing since the last 25 years in biennial cycle. While forest cover monitoring in India has generated valuable data on forest cover in time series, its use, mainly because of the scale (1:50,000 at present), has largely remained at the broad policy and planning level only.

In India, forests are largely State owned and are managed by the State Forest Departments (SFDs). The system of forest administration and management is well established and is in existence for over a century in many areas. Every forest area is under the jurisdiction of a hierarchy of officials of the Forest Department, at the bottom of which is Forest Guard who may be responsible for watching and protecting a forest area ranging from 5 to $30 \mathrm{~km}^{2}$ varying from State to State.

Patrolling of forests and ground verification are essential activities for protecting forests but due to vastness of forest spread and shortage of field staff for protection duties often surveillance of forests is much below the desired level. With the limitations, it is possible that the forest areas which are under damage remain undetected for quite some time. Forest surveillance efforts can be made objective and efficient with the help of technology. The method presented in this paper is in this context of technological intervention for making the field inspections or patrolling of forest areas objective and rational and thus making the whole effort of field monitoring time and cost efficient. More over it provides for a complete scan of the forest area and thus no noticeable change can go undetected. Remote sensing based monitoring should not be seen as an alternative to field visits or ground patrolling but as a tool for making the ground vigil more potent by making it focused and objective and thus making the best use of the personnel who are often much lesser in number than the required strength. Technology based surveillance is unbiased and transparent, if it is blended with the time evolved conventional practices in appropriate way, we can see much higher level of efficiency and effectiveness in the efforts.

\section{Change Detection Using Remote Sensing: The Conventional Approaches}

In the remotely sensed images we get synoptic coverage of an area for different dates and by analyzing the image data set of different dates we can detect changes [2]. The synoptic record of an area in the form of remotely sensed data, not only gives a pictorial view of the area but in the reflectance values in different bands (wavelength ranges) we also get data to analyze the biophysical properties of the land features. According to the conventional approaches of change detection using remote sensing, we either do on-line visual comparison of the two images of different periods and create change polygons, or carry out post classification comparison for generating change maps using automated algorithms [3], there could also be hybrid methods combining the elements of the two. The above two approaches are useful but they have some limitations and may not be appropriate for every intended application, particularly when operational use of change detection is required for vast areas as a monitoring system. Some of the common problems faced in the above remote sensing based change detection methods are as follows:

- Even slight shift in the two images due to imageto-image registration, which is often the case, may cause large number of erroneous change polygons;

- Small change in the radiometry of the two data sets leads to errors;

- Change image results in large number of pixels/polygons;

- Quantifying degree of change is tedious and subjective and therefore prioritizing the change areas is also difficult;

- Handling of large number of change pixels becomes cumbersome;

- Requires a relatively higher level of technical proficiency of the analyst;

- The results may be influenced by the subjectivity and bias of the analyst.

\section{The New Approach}

The new approach presented in this paper for forest monitoring is based on a methodology which makes use of the concept of NDVI and one of the tools of geospatial software for extracting total, mean (and other parameters) of pixel values of a raster image for the polygons of a vector coverage overlaid on it, called 'zonal statistics' in the software parlance.

In this method, as usual we use two images of an area selecting the desired dates (years) of acquisition, also ensuring the same season or month. NDVI transformation is then run on the two images using the digital image processing software. Again with the help of software, a grid mesh (fish net) over the area is created with a pre 
decided grid size. Grid size is to be decided based on the resolution of the satellite data and the unit ground area for which changes are to be monitored, say $500 \mathrm{~m} \times 500$ m (25 ha) or $1 \mathrm{~km} \times 1 \mathrm{~km}$ (100 ha) with IRS P6 LISS III data. A grid should include sufficiently large number of pixels for providing robustness to the index value and at the same time its size should be such that the ground verification could be done in a practicable way. The steps of the methodology are given as follows:

In the Zonal Statistics based approach presented in this study, comparison of NDVI of the two images has been done for grid polygons of uniform sizes (say $500 \mathrm{~m} \times$ $500 \mathrm{~m}$ - $25 \mathrm{ha}$ ) which have been created using "fishnet" feature of the Arc GIS software.

- NDVI images of the two periods are generated.

- Zonal statistics of mean NDVI of all the grids for both the images are generated.

- Mean values of NDVI of the two images are stretched between 0 and 1using global minimum and global maximum values (for uniformity and standardized application for comparing two different dates of images).

- It was observed that stretching of NDVI values between 0 and 1 minimizes the changes due to difference in radiometry of the two images which may arise on account of various atmospheric, weather and sensor related factors.

- Using the above mean and stretched values of NDVI (transformed NDVI) for each grid, an index is derived which gives the percent change in the transformed NDVI values between the corresponding grids.

- The index which may be termed as Grid Vegetation Change index (GVCI) can be mathematically expressed as follows.

A "grid vegetation change index (GVCI)" $I_{j}$ has been defined as the percentage change in the mean values of NDVI for each grid which can be derived by the following formula

$$
I_{j}=100 \times\left(\left(N D V I_{j t 2}^{M^{\prime}}-N D V I_{j t 1}^{M^{\prime}}\right) / N D V I_{j t 1}^{M^{\prime}}\right)
$$

where $N D V I_{j t 1}^{M}$ and $N D V I_{j t 2}^{M}$ are the mean values of $N D V I$ of $j^{\text {th }}$ grid at time $t 1$ and $t 2$ respectively

$$
N D V I_{j}^{M}=\sum_{i} N D V I_{j i} / n
$$

where $N D V I_{j i}$ is the $N D V I$ value of $i^{\text {th }}$ pixel in $j^{\text {th }}$ grid and $\mathrm{n}$ is the total number of pixels in the grid

$N D V I^{M^{\prime}}$ is the stretched value of $N D V I^{M}$ between 0 and 1 taking "global max" and "global min" values of $N D V I^{M}$ of the study area into account.

$$
N D V I^{M^{\prime}}=\frac{N D V I^{M}-\left(N D V I^{M}\right)_{\text {global min }}}{\left(N D V I^{M}\right)_{\text {global max }}-\left(N D V I^{M}\right)_{\text {global min }}}
$$

The above defined Grid Vegetation Change Index (GVCI) is an operational index which significance in terms of biophysical properties has not been studied. This is expected to reflect percentage change in the average NDVI values of a grid between two dates and thus would be correlated to the physical significance of $N D V I$ in a general way.

\section{Work-Flow}

The work-flow is shown in Figure 1.

\subsection{Advantages of the Zonal Statistics Based Approach}

- Provides a robust methodology for identifying areas of forest change between two dates for vast landscapes in a short time;

- An automated approach, free from subjectivity of the interpreter;

- Numerical value of the index gives clear indication of the degree of change;

- Error due to image registration is minimized;

- Impact of radiometric differences in the images due to atmospheric or other factors in identifying change is also minimized;

- Change is given in terms of numerical values of the index (GVCI);

- Gives a complete scan of the area, it is normally not possible that a change above certain threshold goes undetected;

- Change areas can be prioritized using the numerical value of the index for the desired action on the ground.

\subsection{What Makes the Approach Robust?}

- The index is based on DN values of many pixels (more than 100) and therefore the average of NDVI in a grid (say of size $25 \mathrm{ha}$ ) gives a robust parameter about the amount and health of vegetation in that small area.

- With ratio operating at three stages in the index (i.e. NDVI, stretch and percentage), atmospheric and radiometric factors of multiplicative nature which are the potential source of errors get minimized.

- The index also involves minus operation and thus minimizes errors of additive nature.

- The unit area (of observation) becomes an average of many individual pixels and therefore geometric error of pixel level in image registration has lesser consequence in identifying change in the unit area.

\subsection{Unit Area of Observation (Grid)}

Size of unit area of observation i.e. a grid in this methodology may be chosen considering the objectives of monitoring and the pixel size of the satellite data being used. Each grid is identifiable by a unique Id. The size 


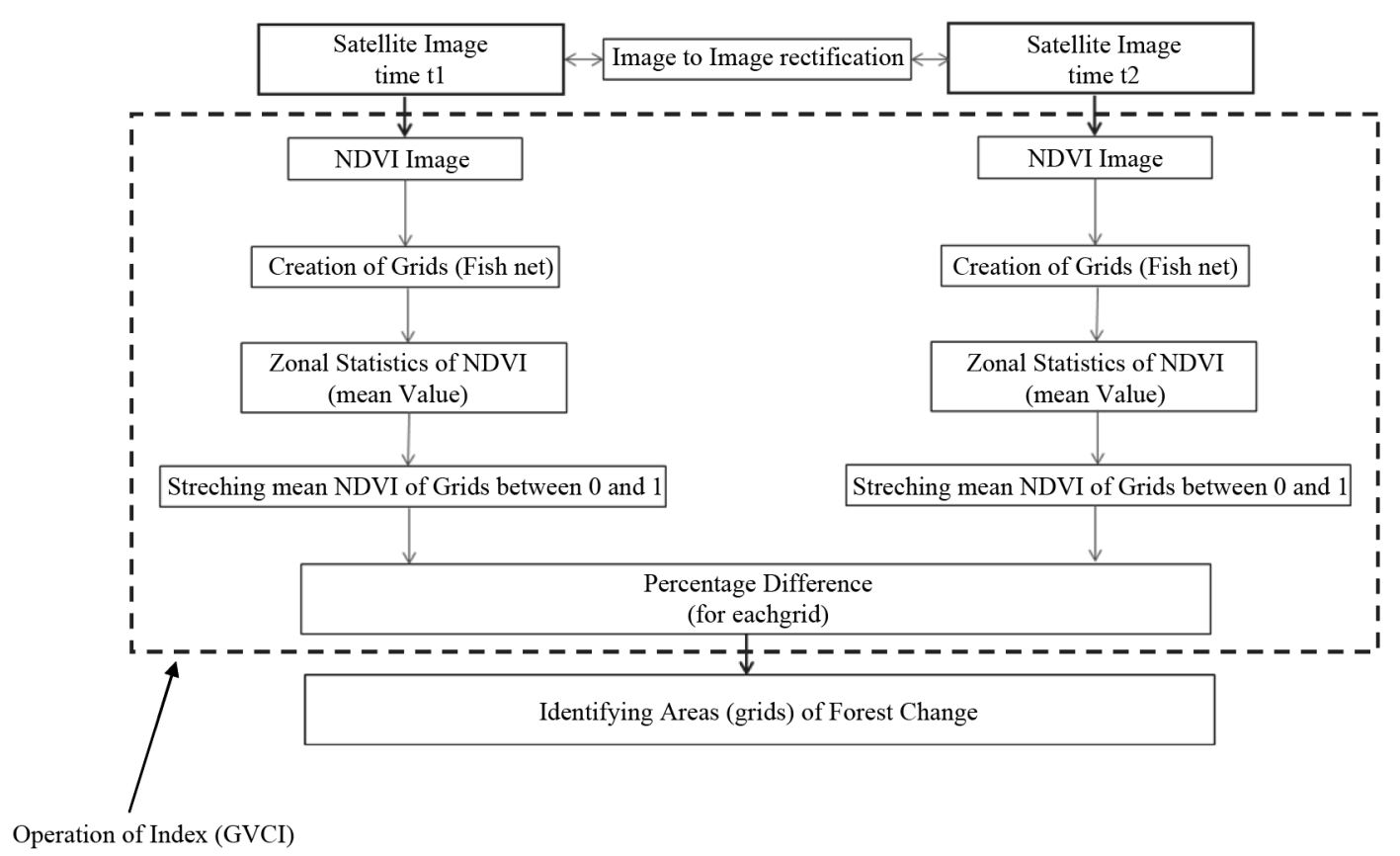

Figure 1. Work-flow of the methodology.

has an implication on field inspection, the grid size should be such that it allows field inspection of the identified grid practicable in a reasonable time e.g. it may be practically feasible to carry out field inspection of a grid of 25 ha in 3 to 4 hours. For intensive monitoring, the grid size may be even smaller like 5 ha or less. Another consideration for choosing the grid size is the resolution of satellite image. Though there is no scientific basis to determine optimum grid size based on spatial resolution of satellite data for GVCI and perhaps it may not be required also, a grid size equivalent to 100 pixels of the satellite data may give enough robustness to the index GVCI. Going by this norm, appropriate grid size in respect of IRS P6 LISS III data of $23.5 \mathrm{~m}$ resolution may be 5.5 ha (i.e. $235 \mathrm{~m} \times 235 \mathrm{~m}$ ) and for IRS P6 LISSIV data of $5.8 \mathrm{~m}$ resolution would be $0.35 \mathrm{ha}(58 \mathrm{~m} \times 58 \mathrm{~m})$.

\section{Illustration}

\subsection{A Vast Area (Largely of Uttarakhand, India) for Change Detection between 1998 and 2010}

The area for illustration of the methodology lies between $77.40^{\circ} \mathrm{E}$ to $79.00^{\circ} \mathrm{E}$ longitude and $29.63^{\circ} \mathrm{N}$ to $30.86^{\circ} \mathrm{N}$ latitude. The expanse of area is $152 \mathrm{~km} \times 142 \mathrm{~km}$ which is nearly 21,500 $\mathrm{km}^{2}$ (Figure 2).

\subsection{Generating NDVI Images of the Two Satellite Images}

NDVI images of the above two satellite images were generated with the help of software (Figure 3).

\subsection{Creation of Fishnet}

A fishnet of $2 \mathrm{~km} \times 2 \mathrm{~km}$ grid size for the whole area was created with the help of GIS software. The whole area comprised 5135 grids. The unit area of change detection in this case becomes a square polygon of $2 \mathrm{~km} \times$ $2 \mathrm{~km}$ which is identifiable by a unique Id, but according to one's choice this can be made $500 \mathrm{~m} \times 500 \mathrm{~m}$ or even smaller (Figure 4).

\subsection{Applying Zonal Statistics Function for Fishnet Vector Coverage over the NDVI Images of the Two Dates}

Zonal statistics function was applied on fishnet vector coverage over the NDVI raster images of the two periods separately (Figure 5).

\subsection{Applying Grid Vegetation Change Index (GVCI) on the Attribute Table}

Grid vegetation change index using the following formula (1) is calculated for each grid and it becomes one of the attributes attached with the grid vector coverage (Table 1).

$$
I_{j}=100 \times\left(\left(N D V I_{j t 2}^{M^{\prime}}-N D V I_{j t 1}^{M^{\prime}}\right) / N D V I_{j t 1}^{M^{\prime}}\right)
$$

\subsection{Selecting the Grids for Different GVCI Values}

Table 1 (as an example) shows the grid Ids, values of GVCI and average number of vegetation pixels in 


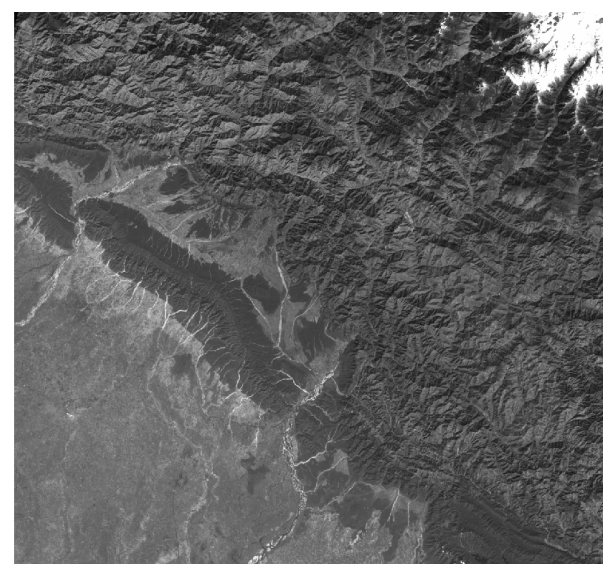

LANDSAT TM Image $12^{\text {th }}$ Nov, 1998

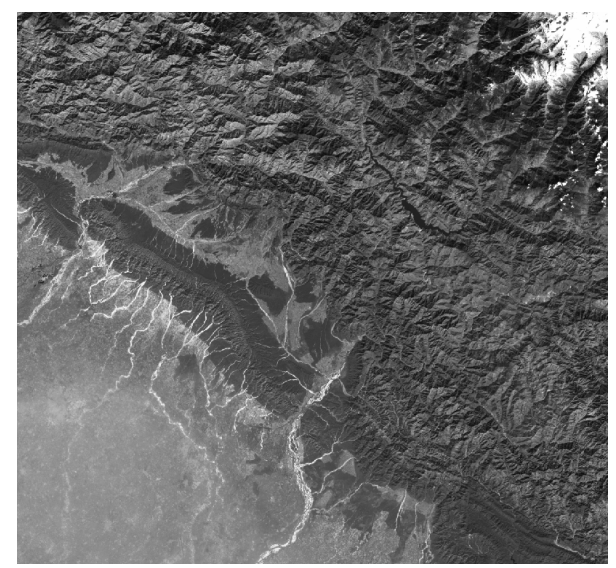

LANDSAT TM Image $12^{\text {th }}$ Nov, 2010

Figure 2. Satellite images of the study area for two dates.

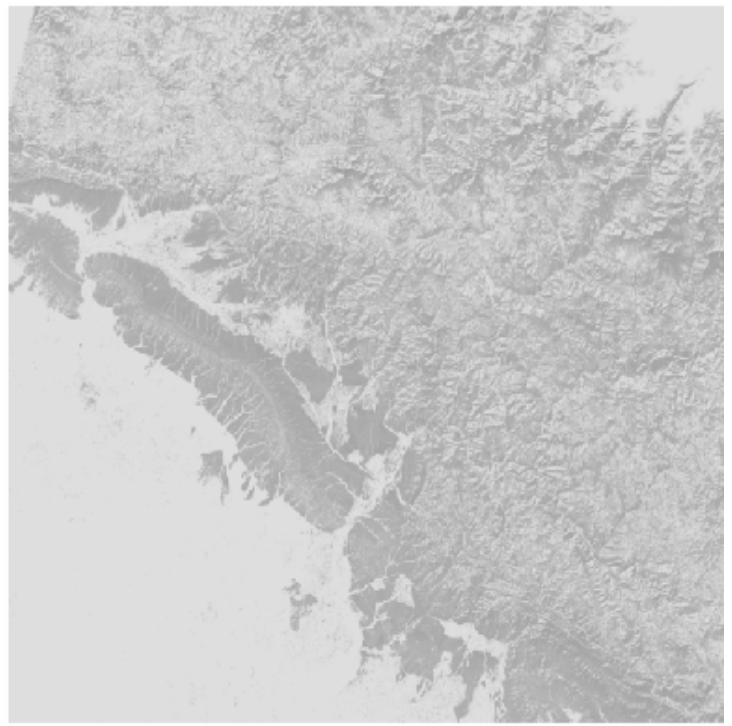

Figure 3. NDVI image of the study area.

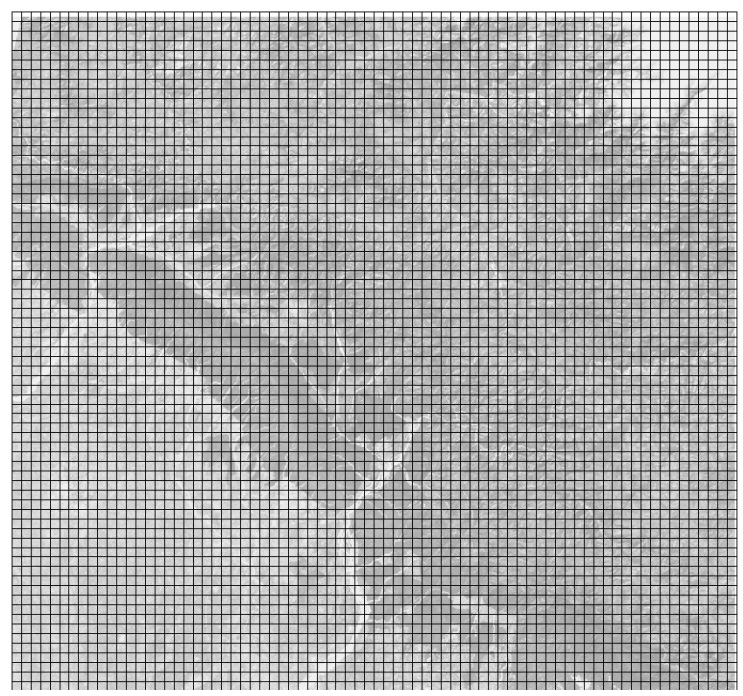

Figure 4. Fishnet over the study area. 

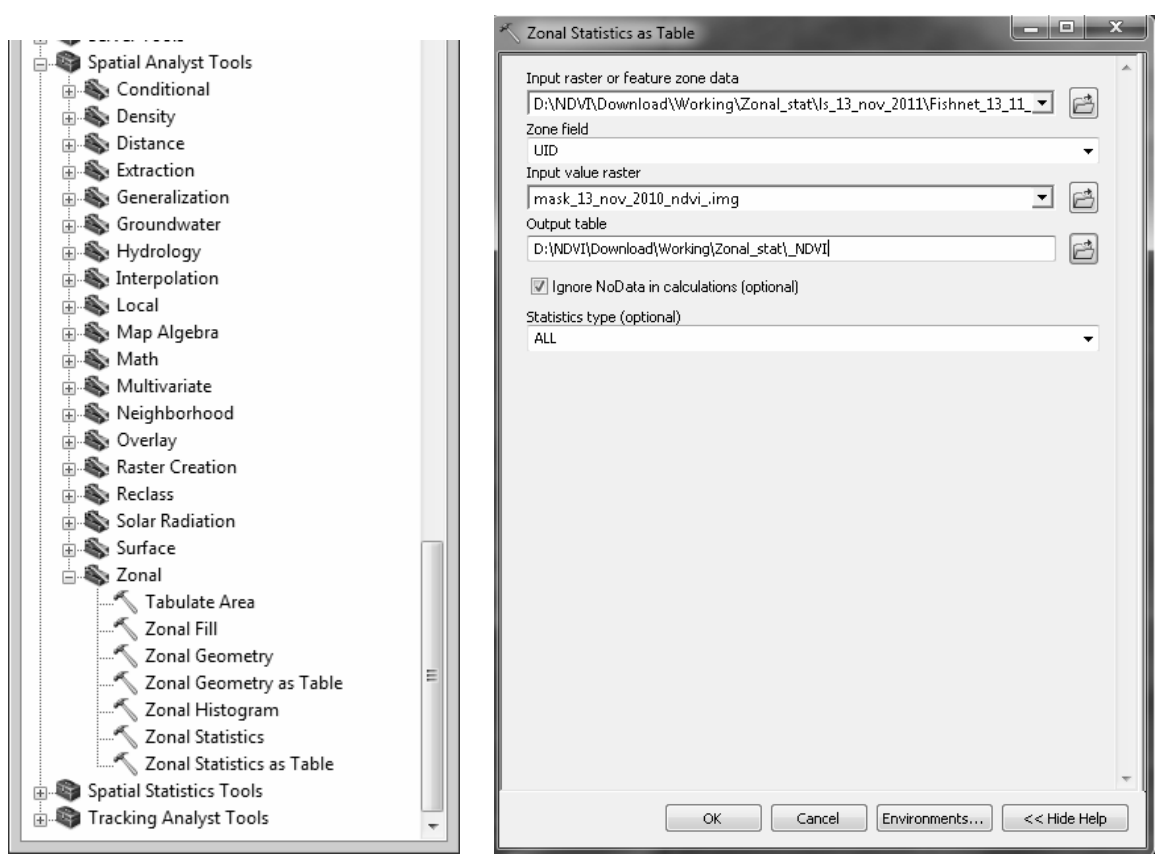

Figure 5. Zonal statistics template.

Table 1. Grid Ids, index values and average sum of vegetation pixels (extent of vegetation in the grid)—part only.

\begin{tabular}{ccc}
\hline Grid_Id & GVCI & Average Sum of Vegetation Pixels \\
\hline 1000 & -1.137 & 155.856 \\
1001 & -4.023 & 147.055 \\
1002 & -1.820 & 154.738 \\
1003 & -1.105 & 152.316 \\
1004 & -1.085 & 141.402 \\
1005 & -0.049 & 152.935 \\
1006 & -1.471 & 154.213 \\
1007 & -1.108 & 143.689 \\
1008 & 0.446 & 145.764 \\
1009 & -10.089 & 144.521 \\
1010 & -10.571 & 130.026 \\
1011 & -10.749 & 108.769 \\
1012 & -21.669 & 25.909 \\
1013 & -5.073 & 6.605 \\
1014 & -8.450 & 24.498 \\
1015 & -1.839 & 159.181 \\
1016 & -1.311 & 144.523 \\
1017 & 2.261 & 150.984 \\
1018 & 4.087 & 153.793 \\
1019 & 4.443 & 145.125 \\
1020 & 5.005 & 153.985 \\
contd. & & \\
\hline & &
\end{tabular}

the grids i.e. the extent of vegetation cover. The grid Id 1012 shows the maximum change which is negative but the grid has low area under vegetation. The grids with Ids 1009 and 1010 also show significant negative change and have good extent of vegetation. The grid Id 1020 shows positive change (improvement) and has good vegetation cover. Selection of grids and display of change on the computer screen based on the criteria of one's choice can be easily done with the GIS software. Product of the GVCI and "average sum of vegetation pixels" will give 
an indicative measure of total vegetation change in a grid. The total of the product for all the grids will give a measure of total vegetation change in a given study area.

The change analysis of the above area using $2 \mathrm{~km} \times 2$ $\mathrm{km}$ grids has shown number of grids in different degree of vegetation change in terms of the GVCI values. The negative changes or losses is shown by the negative values, whereas, the gains or positive changes in the vegetation are shown by the positive values of GVCI (Table 1).

Table 2 shows that out of total 5395 grids in the study area, $4016(74.4 \%)$ grids show loss in vegetation during the period 1998 and 2010 and only 1379 (25.6\%) grids show gain in vegetation. Thus the area selected for illustration shows over all negative change in the vegetation.

As illustrated above one can use GVCI values in selecting the "candidate grids with significant changes" which may be above (or lower in case of negative changes) certain threshold value of GVCI. Such grids may then be field inspected for ascertaining ground details and studying the reasons of change.

\subsection{Visualization of Changes in the Grids Selected by GVCI Values}

The above procedure was applied on several pairs of remote sensing Images of different areas and in every case the above methodology effectively highlighted significant change areas on images spanning over areas 5000 to $15,000 \mathrm{~km}^{2}$ (Figure 6).

\section{Limitations}

1) The GVCI works well in highlighting the grids where vegetation change has taken place, though sometimes atmospheric conditions like haze and clouds etc.

Table 2. Summary of GVCI values showing overall changes in the study area.

\begin{tabular}{cc}
\hline GVCI Values & No. of Grids \\
\hline less than -40 & 361 \\
between -40 and -30 & 272 \\
between -30 and -20 & 282 \\
between -20 and -10 & 570 \\
between -10 and 0 & 2531 \\
between 0 and 10 & 1335 \\
between 10 and 20 & 24 \\
between 20 and 30 & 4 \\
between 30 and 40 & 5 \\
Greater than 40 & 11 \\
Total & $\mathbf{5 3 9 5}$ \\
\hline
\end{tabular}
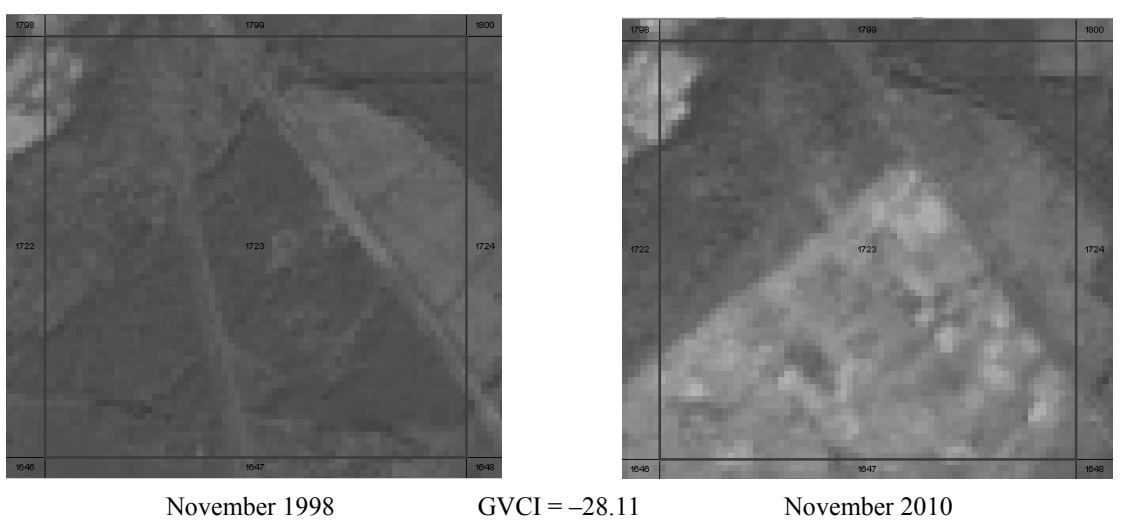

(a)

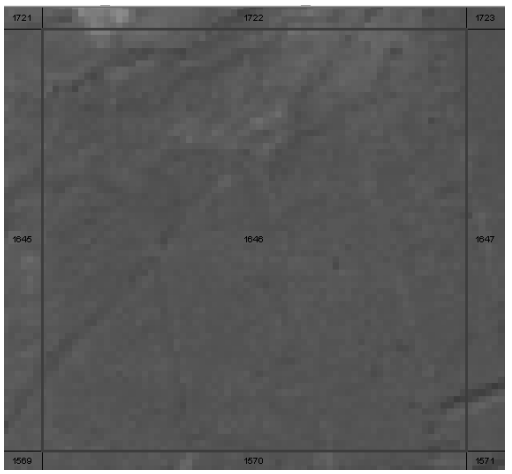

November 1998

$\mathrm{GVCI}=-35.89$

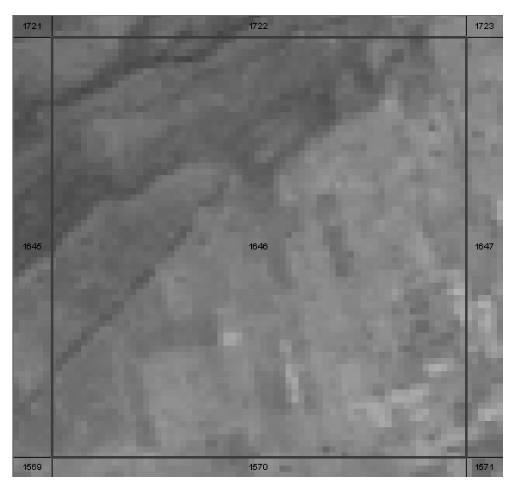

November 2010

(b) 

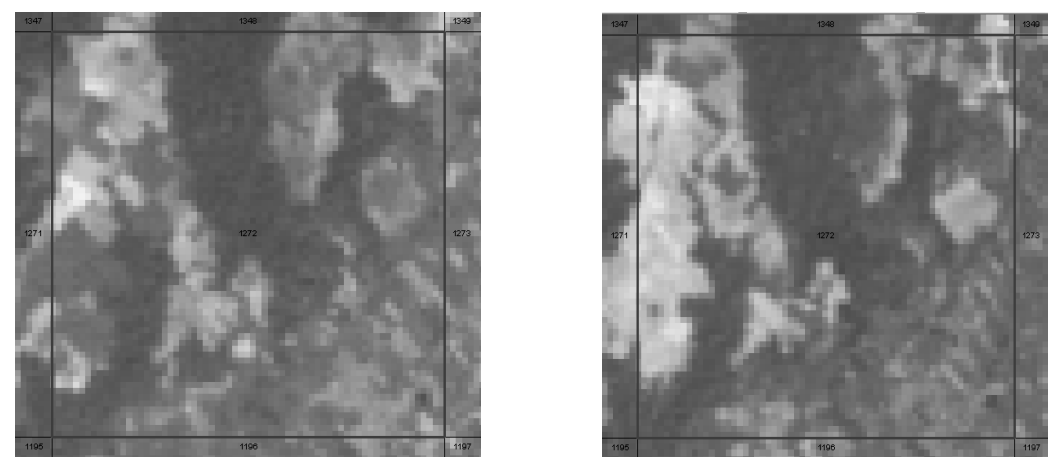

November 1998 GVCI $=-21.91$

November 2010

(c)
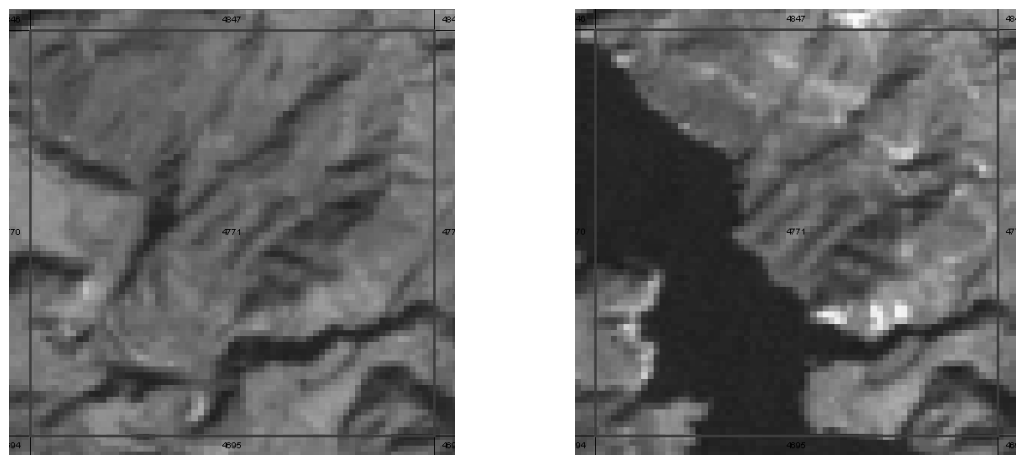

November 1998 $\mathrm{GVCI}=-43.24$

November 2010

(d)
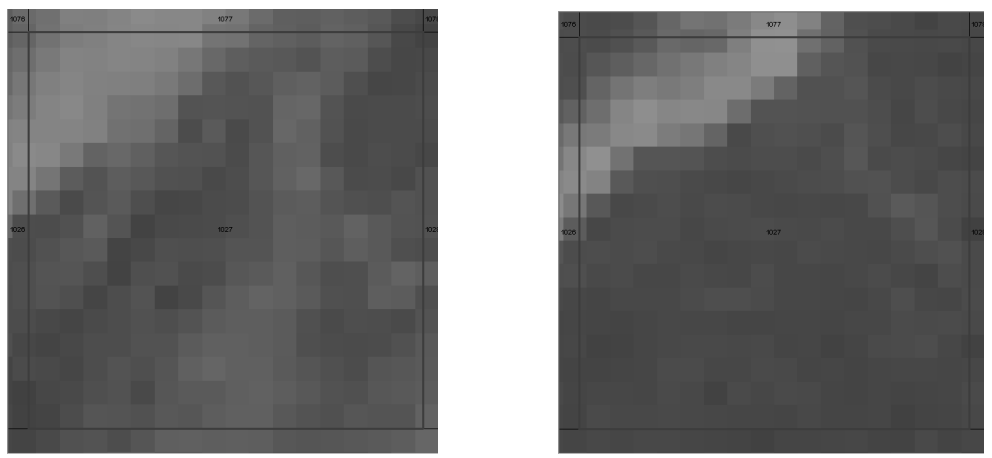

November 1998

$\mathrm{GVCI}=+14.71$

(e)

November 2010

Figure 6. Illustration of changes.

obscure the real change in vegetation and the change highlighted by the index in a grid may be influenced by these atmospheric factors. This limitation can be overcome to a great extent by preprocessing of satellite data for atmospheric correction.

2) Radiometric variations in the satellite images due to changes in satellite ephemeris, illumination conditions or aging of the satellite/sensor would affect the GVCI values to some extent and thus influence the detection of forest change.

3) Changes in the deep shadow areas are not detected.

4) It is necessary that the two time satellite images used in the change detection by above approach should be from the same season (month of the year).

\section{Conclusion}

The methodology presented in this paper is a new remote sensing based approach for scanning vast landscapes for detecting changes in forests or other vegetation forms in a pointed manner, highlighting small square tiles (say 25 ha or smaller) where change in forest/vegetation has taken place. The paper introduces a new index (GCVI) based on NDVI and a spatial feature of GIS software (computing zonal attributes) for detection of changes in forests. The method can be effectively used in making the ground inspections objective and in identifying sensi- 
tive areas for taking effective measures for arresting the causes of any degradation process. It should not be seen as an alternative to the field patrolling based surveillance of forests, rather this method coupled with field patrolling can make the effort much more effective. Quantification of change by this method can help in prioritizing the areas for intense monitoring. The approach can also be used in monitoring implementation of afforestation and conservation activities under various schemes. This methodology can be practicably implemented in the State Forest Departments with one centralized system monitoring a large forest area on a desired periodicity.

\section{Acknowledgements}

The author gratefully acknowledges support and encouragement given by Director, IGNFA and help rendered by Smt Pushpalatha Devala, Technical Associate. The study was carried out in the Geomatics Lab of IGNFA.

\section{REFERENCES}

[1] Forest Survey of India, India State of Forest Report (ISFR), 2009.

[2] A. J. Elmore, J. F. Mustard, S. J. Manning and D. B. Lobell, "Quantifying Vegetation Change in Semiarid Environments: Precision and Accuracy of Spectral Mixture Analysis and the Normalized Difference Vegetation Index," Remote Sensing of Environment, Vol. 73, No. 1, 2000, pp. 87-102.

[3] J. G. Lyon, D. Yuan, R. S. Lunetta and C. D. Elvidge, "A Change Detection Experiment Using Vegetation Indices," Photogrammetric Engineering \& Remote Sensing, Vol. 64, No. 2, 1998, pp. 143-150. 